\title{
Detecting nanometric displacements with optical ruler metrology
}

\author{
Guang Hui Yuan ${ }^{1}$, Nikolay I. Zheludev ${ }^{1,2^{*}}$
}

${ }^{1}$ Centre for Disruptive Photonic Technologies, The Photonics Institute, SPMS, Nanyang Technological University, Singapore 637371, Singapore

${ }^{2}$ Optoelectronics Research Centre and Centre for Photonic Metamaterials, University of Southampton, Highfield, Southampton, SO17 1BJ, UK

*Corresponding author. Email: niz@orc.soton.ac.uk

Abstract: We introduce the optical ruler, an electromagnetic analogue of a physical ruler, for nanoscale displacement metrology. The optical ruler is a complex electromagnetic field in which singularities serve as the marks on the scale. It is created by the diffraction of light on a metasurface, with singularity marks then revealed by high-magnification interferometric observation. Using a Pancharatnam-Berry phase metasurface, we demonstrate a displacement resolving power of better than $1 \mathrm{~nm}(\lambda / 800)$ at a wavelength of $800 \mathrm{~nm}$. We argue that resolving power of $\sim \lambda / 4000$, the typical size of an atom, may be achievable. An optical ruler with dimensions of only a few tens of microns offers applications in nano-metrology, nanomonitoring and nano-fabrication, in particular in the demanding and confined environment of future smart manufacturing tools.

One Sentence Summary: Light field with singularities can replace physical ruler as metrology reference giving access to resolution exceeding one nanometer. 
Light is a perfect tool for metrology as it allows measurements of the size or position of an object against a ruler, in the same way as the Egyptians and Mesopotamians used rods divided into cubits, the earliest known unit of length, in the third millennium BC. Displacement of an object against a ruler can be measured optically with resolution limited by diffraction to about half of the used optical wavelength $\lambda$, typically a fraction of micrometer. In this paper, we demonstrate that orders of magnitude higher resolution, down to the atomic size level can be achieved by using - instead of a physical ruler - an optical ruler: an electromagnetic field structured with singularities at the deeply sub-wavelength scale. This novel metrology is based on the premise that free-space singular optical fields with deeply sub-wavelength zones of rapid phase variations can be created by interference of multiple beams of light (1-10) and that these free-space optical fields can be imaged with no limit to resolution, far beyond the conventional diffraction limit. In contrast with superoscillatory optical microscopy using sub-diffraction intensity hotspots (8), the optical ruler metrology exploits phase singularities that are much stronger localized.

To create an optical ruler, we employ a nanostructured metasurface that, when illuminated with coherent light of wavelength $\lambda$, produces in free-space an optical field with high gradient of phase $\varphi$ where the local wavevector $\boldsymbol{k}_{\text {local }}=\nabla \varphi$ considerably exceeds the free-space wavevector $\left|\boldsymbol{k}_{0}\right|=2 \pi / \lambda$. The interference of this field with the incident wave itself reveals the zones with high values of local wavevectors that can be as small as a fraction of one nanometer in size. We reveal these singularities interferometrically using the same metasurface as a micronscale size monolithic interferometer. We image the singularities with high magnification and use them as the reference points (marks on the scale) for super-resolution metrology in much the same way that marks on the physical ruler are used in conventional measurements (see Fig. 1).

The optical ruler is generated by a planar Pancharatnam-Berry phase metasurface $(11,12)$ illuminated with a semiconductor laser at the wavelength of $\lambda=800 \mathrm{~nm}$ (13). We constructed the metasurface that creates in the far-field from the plane of the metasurface a superoscillatory subwavelength hotspot $(7,8,10)$ with polarization orthogonal to the incident wave. Such hotspots are known to be flanked by zones of high phase gradient (10). We reveal the zones of high phase gradients on the optical ruler by observing the interference between the superoscillatory field and 
the reference wave transmitted through the metasurface with the same polarization as the incident wave.

We image the interference pattern of the optical ruler without any loss of resolution by a lens with a numerical aperture that is higher than that of the pattern generator. We used a $1300 \mathrm{X}$ magnification optical system, projecting the image on an image sensor with pixel size of $6.5 \mu \mathrm{m}$, thus achieving an effective pixel resolution of $5 \mathrm{~nm}$ in the observation plane (13). The experimental data are compared with the patterns simulated by FDTD modelling. From the interferometric intensity maps (Figs. 2A \& 2B) taken at four different incident polarizations $\left(+45^{\circ}\right.$ and $-45^{\circ}$ linear polarization, and right and left circular polarizations), using the technique described in ref. $(10,13)$, we retrieve the intensity (Figs. 2C \& 2F), phase (Figs. 2D \& 2G), and the transverse local wavevector $k_{x}=\nabla_{x} \varphi$ (Figs. $2 \mathrm{E} \& 2 \mathrm{H}$ ) profiles of the superoscillatory field. It appears that the zones of rapid variations of phase (peaks of the local wavevector) are much narrower than the intensity hotspot itself. We used these peaks as "marks" of the optical ruler.

To evaluate the practically achievable resolution of the optical ruler displacement metrology, we moved the platform A holding the laser source and metasurface interferometer in the lateral direction against the image sensor on platform $\mathbf{B}$ with nanometric steps and recorded positions of the marks of the optical ruler, see Fig. 3. The linear regression analysis shows that the dependence of the recorded position of the marks against their physical position has a unitary slope and standard deviation of less than $1 \mathrm{~nm}$. This determines the displacement resolving power of the optical ruler to be better than $\lambda / 800$. Here, the resolving power of $\sim 1 \mathrm{~nm}$ is limited by the resolution of the piezo actuator $(0.4 \mathrm{~nm})$, mechanical instability in the setup, and pixilation of the image sensor. To evaluate the potential resolution of the metrology, we calculated the autocorrelation function of the experimentally measured and simulated intensity map $G_{I}\left(\delta_{x}\right)=\int I\left(x+\delta_{x}\right) I(x) d x$ and the wavevector map $G_{k}\left(\delta_{x}\right)=\int k\left(x+\delta_{x}\right) k(x) d x$ taking into account only $k(x)>2$. Such autocorrelation functions allow a universal estimate to how well the original and displaced images can be distinguished, while the resolving power is evaluated as width of the autocorrelation function at $80 \%$ of its height. The profile of the autocorrelation function $G_{I}\left(\delta_{x}\right)$ appears to be bell-shaped, giving a resolution of $249 \mathrm{~nm}(0.31 \lambda)$ 
from the computational data (Figs. 3C \& 3D), and $269 \mathrm{~nm}(0.34 \lambda)$ from the empirically measured intensity map (Figs. 3G \& 3H). A much better resolving power can be achieved by using a $k$-vector map (Figs. 3E \& 3I): its autocorrelation function $G_{k}\left(\delta_{x}\right)$ decays rapidly and is exponential at small distances giving a resolution of about $190 \mathrm{pm}(\lambda / 4200)$ as estimated from the simulated data, and $1.7 \mathrm{~nm}(\lambda / 470)$ as evaluated from the empirically measured intensity maps (Figs. 3F \& 3J), which is close to the value of standard deviation obtained in the displacement measurements (Figs. 3A \& 3B).

We have also demonstrated two-dimensional optical ruler metrology with a random twodimensional Pancharatnam-Berry phase metasurface (Fig. 4). The phase recovery process reveals a complex phase map with a high density of singularities (Fig. 4C) and superoscillatory wavevectors (Fig.4D). The autocorrelation function $G_{k}\left(\delta_{x} ; \delta_{y}\right)=\iint k\left(x+\delta_{x}, y\right) k\left(x, y+\delta_{y}\right) d x d y$ decays rapidly on the nanometer scale and is exponential at small distances allowing for a displacement resolution of about $2.2 \mathrm{~nm}(\lambda / 360)$. Lower resolution of the two-dimensional optical ruler in comparison with the one-dimensional case is explainable by the use of a superoscillatory generator creating a pattern of singularities of diverse width.

A radical improvement of displacement resolving power from observing the intensity maps to observing the $k$-vector maps relates not only to a dramatic difference in the widths of their "marks". It is also helped by the different nature of the intensity and $k$-vector peak profiles that is evident from their autocorrelation functions: the smooth bell-shaped $G_{I}\left(\delta_{x}\right)$ falls much slower than that of the exponentially localized $G_{k}\left(\delta_{x}\right)$.

Our results show that with the optical ruler, a displacement resolving power of about $\lambda / 4000$ (e.g. $100 \mathrm{pm}$ at $\lambda=400 \mathrm{~nm}$ ) could be potentially achievable, bringing it to the true atomic scale. Areas of large wavevectors are characteristic to many optical fields, however narrower peaks require monochromatic light and tend to be located in areas of lower intensity $(3-5,14)$. Moreover, high resolution will require filed mapping with high magnification, which reduces light's intensity on the sensor. Therefore, the finite spectrum of the light source and noise level at the detector will limit the resolution. Our modelling shows that an increase of the noise level at the detector from 
$0 \%$ to $20 \%$ results in a steady decrease of resolving power from $190 \mathrm{pm}$ to $1.5 \mathrm{~nm}$ respectively (13). Above all, a demonstration of the resolving power at the level of $\lambda / 4000$ will require the use of ultra-stable opto-mechanics similar to that used in atomic resolution STM instruments, and a further increase of optical magnification of the imaging system to reduce pixilation.

In summary, we demonstrate a novel displacement nanometrology based on the observation of optical singularities which supersedes the conventional diffraction limit of resolution for displacement measurements with a physical ruler by direct observation by several orders of magnitude. Similar to STED, PALM and STORM, the proposed approach is also a far-field technique and therefore allows for non-contact operation. The sub-nanometer resolving power of the optical ruler demonstrated here is higher than that of other optical super-resolution techniques. It does not require the high intensities for STED bleaching, or the data accumulation required for PALM and STORM. However, the optical ruler technique does not allow full image reconstruction and is only suitable for metrology. Additionally, it does not suffer from the mechanical and thermal instabilities, which affect large conventional metrological interferometric instruments. The metasurface can be manufactured at the tip of an optical fiber allowing numerous applications where high resolution, small size and non-contact operation are essential including monitoring displacements of scanning stages of AFM, STM, and superresolution optical microscopes, lithography mask alignment, and the control of motion of tools in nano-assembly. The optical ruler can also be placed on nano-indenter heads to measure the modulus of elasticity, yield stress, hardness, and wear resistance of materials. One can even envisage the optical ruler attached to a cutting tool of a smart manufacturing lathe or milling machine. Lastly, the optical ruler can be used for monitoring of mutual nanoscale displacements of parts of precision constructions, such as large optical telescopes, disc drives, MEMS/NEMS devices, acceleration sensors, and for monitoring the deformation, fatigue or thermal expansions of components.

\section{References and Notes:}

1. M. S. Soskin, M. V. Vasnetsov, Singular optics. Prog. Opt. 42, 219-276 (2001).

2. J. Leach, M. R. Dennis, J. Courtial, M. J. Padget, Knotted threads of darkness. Nature 432, 165 (2004).

3. M. R. Dennis, K. O’Holleran, M. J. Padgett, Singular optics: Optical vortices and polarization singularities. Prog. Opt. 53, 293-363 (2009). 
4. M. V. Berry, M. R. Dennis, Knotted and linked phase singularities in monochromatic waves. Proc. R. Soc. Lond. A 457, 2251-2263 (2001).

5. M. V. Berry, S. Popescu, Evolution of quantum superoscillations and optical superresolution without evanescent waves. J. Phys. A Math. Gen. 39, 6965-6977 (2006).

6. M. R. Dennis, A. C. Hamilton, J. Courtial, Superoscillations in speckle patterns. Opt. Lett. 33, 2976-2978 (2008).

7. F. M. Huang, N. I. Zheludev, Super-resolution without evanescent waves. Nano Lett. 9, 1249-1254 (2009).

8. E. T. F. Rogers, N. I. Zheludev, Optical super-oscillations: sub-wavelength light focusing and super-resolution imaging. J. Opt. 15, 094008 (2013).

9. M. V. Berry, Quantum backflow, negative kinetic energy, and optical retro-propagation. $J$. Phys. A Math. Theor. 43, 415302 (2010).

10. G. H. Yuan, E. T. F. Rogers, N. I. Zheludev, "Plasmonics" in free space: observation of giant wavevectors, vortices and energy backflow in superoscillatory optical fields. Light Sci. Appl. 8, 2 (2019).

11. Z. Bomzon, G. Biener, V. Kleiner, E. Hasman, Space-variant Pancharatnam-Berry phase optical elements with computer-generated subwavelength gratings. Opt. Lett. 27, 1141-1143 (2012).

12. D. Lin, P. Fan, E. Hasman, M. L. Brongersma, Dielectric gradient metasurface optical elements. Science 345, 298-302 (2014).

13. Supplementary Materials.

14. M. R. Dennis, J. Lindberg, Natural superoscillation of random functions in one and more dimensions. Proc. SPIE 7394, 73940A (2009).

15. The data from this paper can be obtained from the University of Southampton research repository: https://doi.org/10.5258/SOTON/xxxx.

16. E. T. F. Rogers, J. Lindberg, T. Roy, S. Savo, J. E. Chad, M. R. Dennis, N. I. Zheludev, A super-oscillatory lens optical microscope for subwavelength imaging. Nat. Mater. 11, 432 (2013).

Acknowledgments:

Funding: This work was supported by the Singapore Ministry of Education (Grant MOE2016T3-1-006), ASTAR QTE Programme (Grant SERC A1685b0005), the Engineering and Physical Sciences Research Council UK (Grant EP/N00762X/1). Author contributions: N.I.Z. conceived the idea of nanometrology using optical superoscillations. G.H.Y. designed the monolithic super-oscillatory metasurface, and performed the fabrication, measurement and data analysis. N.I.Z. and G.H.Y. wrote the paper, discussed the results and cross-edited the manuscript. N.I.Z. supervised and coordinated all the works. Competing interests: Authors declare no competing interests. Data and materials availability: All data are available in the manuscript, supplementary material, and the repository (15). 


\section{Supplementary Materials:}

Supplementary Text

Figures S1-S2

References (16)

\section{Figure Legends:}

Fig. 1. Principle of the optical ruler displacement metrology. Conventional displacement metrology is based on the observation of a physical ruler moving along the MM line. The optical ruler metrology relies on observing the deeply sub-wavelength zones of a complex optical field with strong phase gradient $\boldsymbol{k}_{\text {local }}=\nabla \varphi$ along the MM line, that are used as "marks" of the optical ruler.

Fig. 2. Intensity, phase and local wavevector values along the optical ruler. (A, B) The $x-y$ cross-section of intensity distribution of the $y$-polarized component of the optical field created by the metasurface at different incident polarizations $\left(x,+45^{\circ} / 45^{\circ}\right.$ linear polarization, and right/left circular polarization $\mathrm{RCP} / \mathrm{LCP}$ ), at the distance of $\sim 10 \mu \mathrm{m}$ from the metasurface. (A) FDTD simulation; (B) Experimental data. Dashed horizontal bar indicates interval of $2 \mu \mathrm{m}$ on all images. (C, F) Intensity profiles for different input polarizations (insets show profiles as taken by the camera). (D, G) Retrieved phase profiles. $(\mathbf{E}, \mathbf{H})$ Local wavevector $k_{x}=\nabla_{x} \varphi$ exhibits peaks localized at the nanoscale (dashed red lines show $\left|k_{x}\right| / k_{0}=1$ ). (I) SEM of the fragment of a 1D Pancharatnam-Berry phase metasurface that creates the superoscillatory field with zones of high phase gradients. Artificial colours in the zoom-in indicate columns providing either 0 or $\pi$ phase shift in the transmitted light. 
Fig. 3. Demonstration of the optical ruler displacement sensing. (A) Positions of the optical ruler marks (wavevector peaks) marked by red upward triangles and magenta downward triangles and their mean values (black circles) measured at different positions of platform A with respect to platform B with steps of $3 \mathrm{~nm}$. (B) Positions of the marks (wavevector peaks) measured with steps of $1 \mathrm{~nm}$. Details of the field maps of the optical ruler are presented on the plates $(\mathbf{C}-\mathbf{J})$ : $(\mathbf{C}, \mathbf{G})$ The $x-y$ cross-sections of intensity distribution of the $y$-polarized complex optical field component created by the metasurface at the distance of $\sim 10 \mu \mathrm{m}$ from the metasurface, $(\mathbf{D}, \mathbf{H})$ their autocorrelation functions, (E,I) $x$ - $y$ cross-sections of $k$-vector maps, and $(\mathbf{F}, \mathbf{J})$ their autocorrelation functions.

Fig. 4. Two-dimensional optical ruler. (A) SEM image of the fragment of a $2 \mathrm{D}$ random Pancharatnam-Berry phase metasurface; $(\mathbf{B}, \mathbf{C}) x-y$ intensity $(\mathrm{B})$ and phase $(\mathrm{C})$ maps in free space at $\sim 10 \mu \mathrm{m}$ above the metasurface illuminated by coherent light source at the wavelength $\lambda=800$ nm; black dots show phase singularity points; (D) Retrieved wavevector map; (E,F) Crosssections of the autocorrelation function along $x$ - and $y$-direction of the wavevector map. 

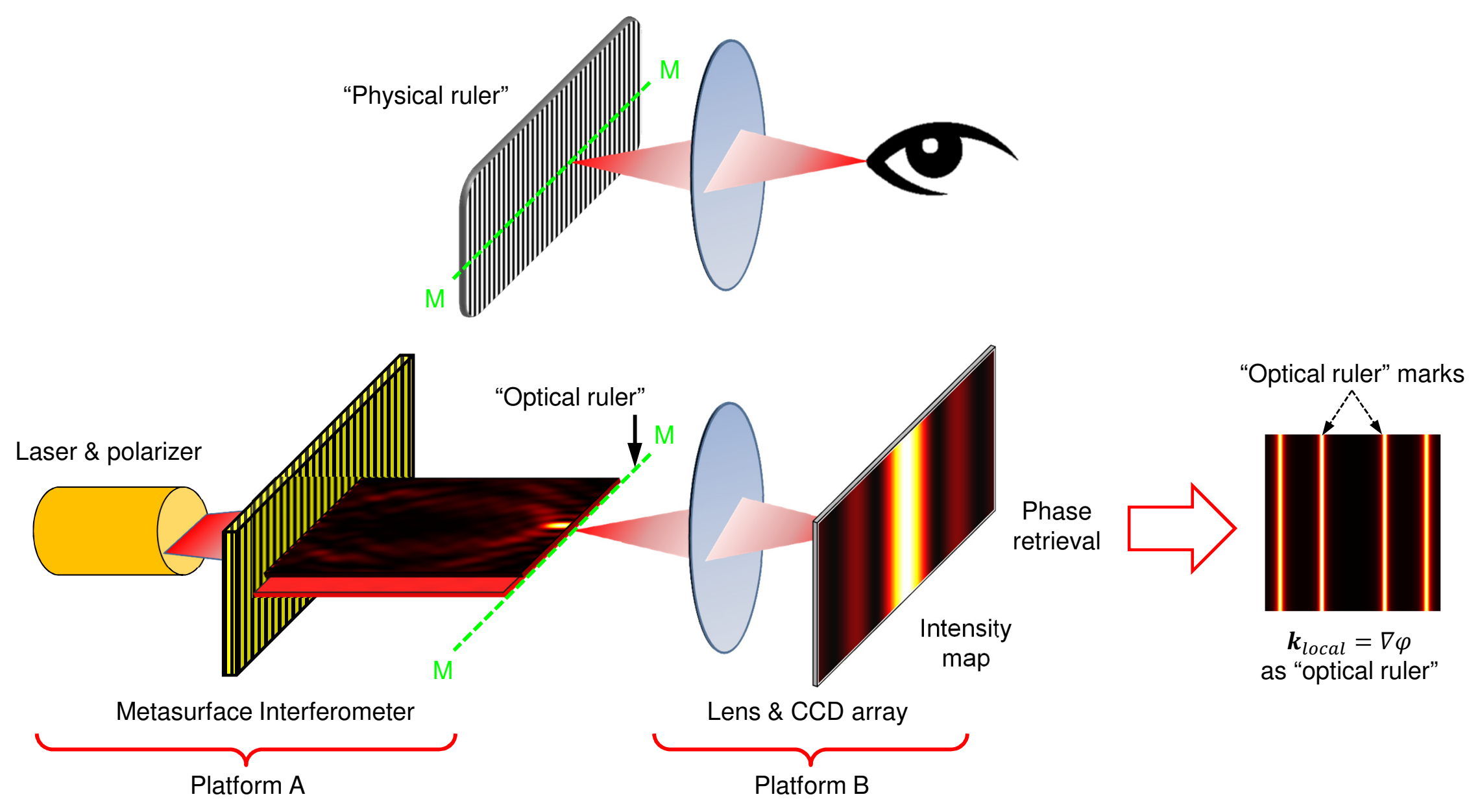


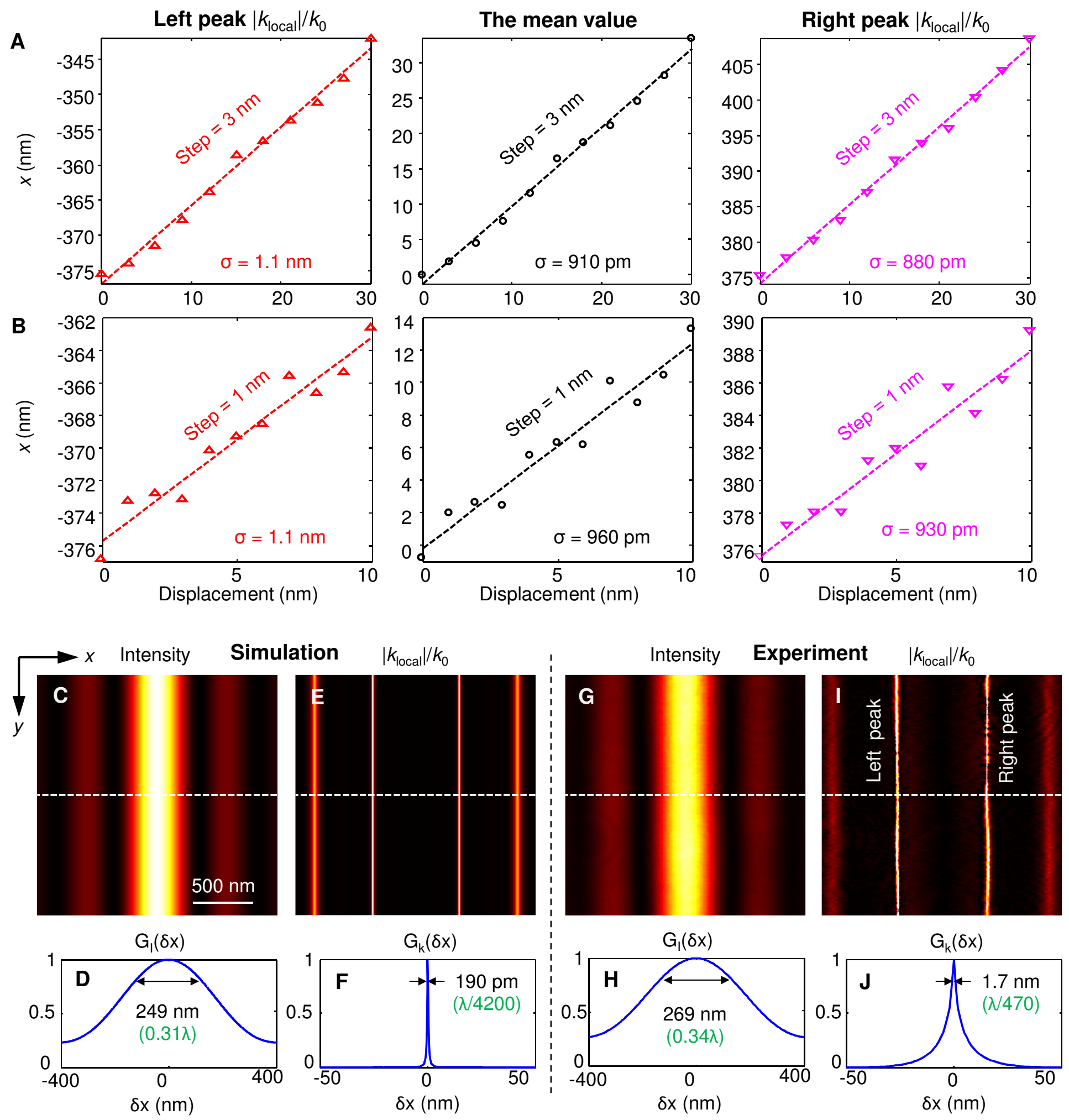


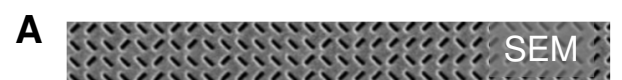

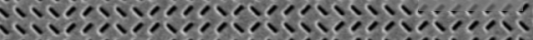

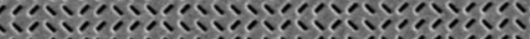

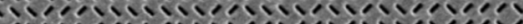

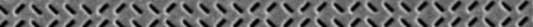

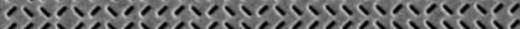

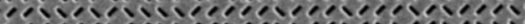
 

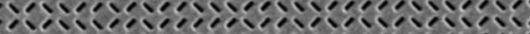

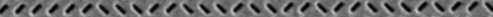

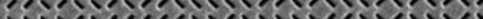
促e

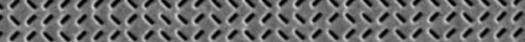

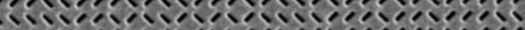

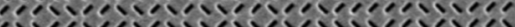


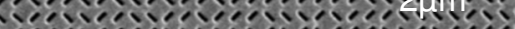

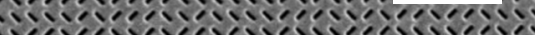

D

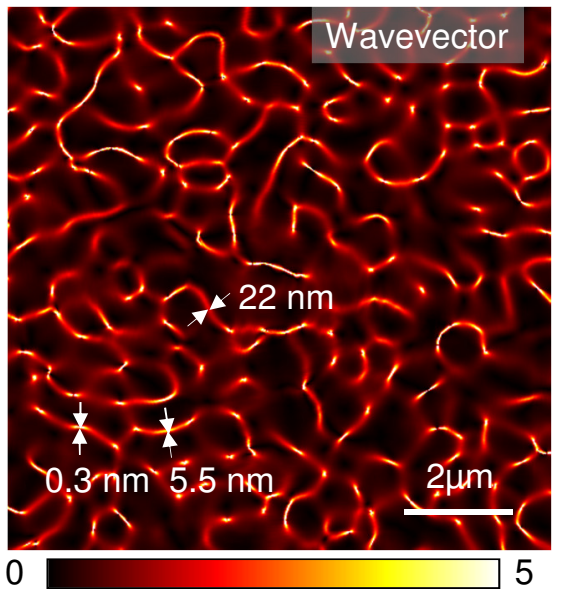

B

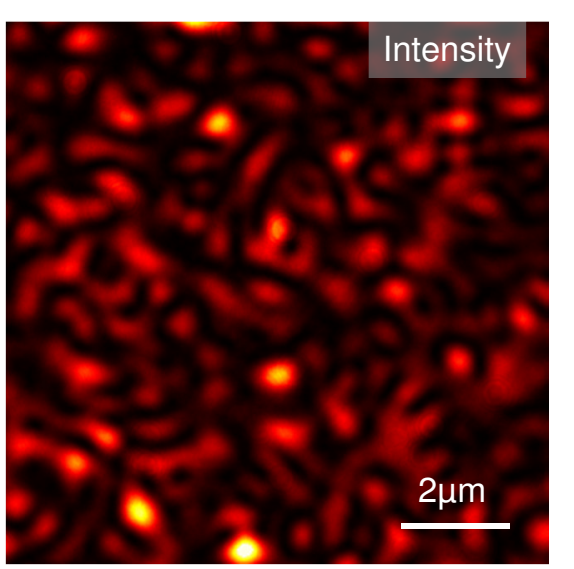

E

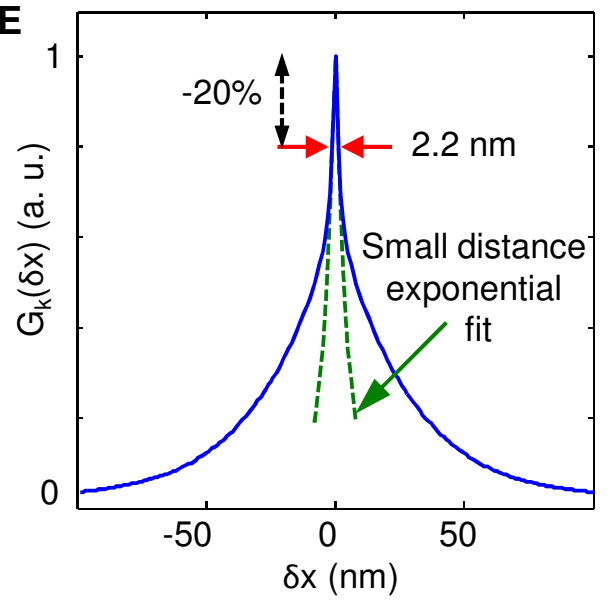

C

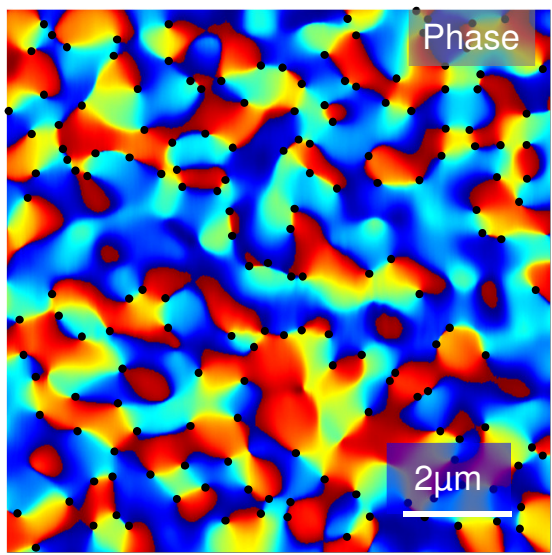

F

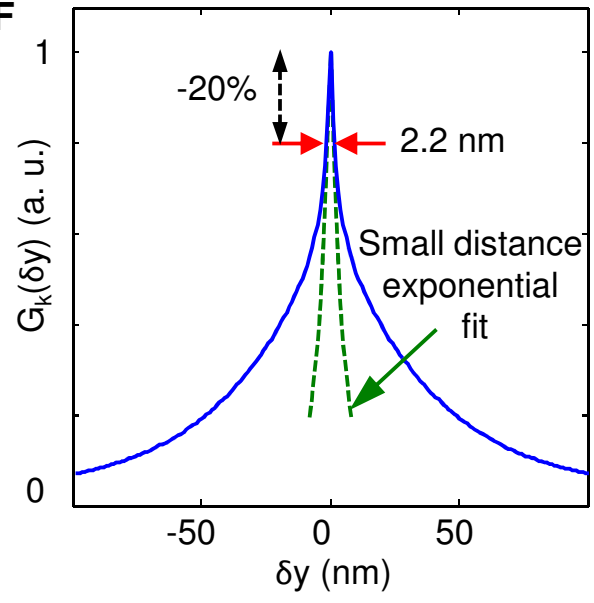




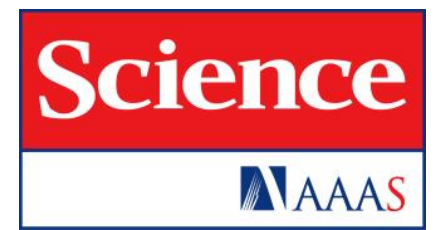

\title{
Supplementary Materials for
}

\section{Detecting nanometric displacements with optical ruler metrology}

\author{
Guang Hui Yuan, Nikolay I. Zheludev* \\ *Corresponding author. Email: niz@orc.soton.ac.uk
}

This PDF file includes:

Supplementary Text

Figs. S1 to S2

References 


\section{Supplementary Text}

\section{Experimental setup}

Detailed experimental setup is given in Fig. S1. The laser source is a diode laser with emission wavelength of $800 \mathrm{~nm}$ (Toptica DLC DL pro 780) and line-width of $100 \mathrm{kHz}$. A linear polarizer (P1) is used to purify the incident polarization, followed by a quarter waveplate (QWP) or half waveplate (HWP) to achieve the desired incident polarization ( $x, y,+45^{\circ},-45^{\circ}$ linear polarizations, and left and right circular polarizations). The field distribution created by the metasurface was mapped with a high-resolution camera (Andor Neo sCMOS, 2560*2160 pixels, pixel size $6.5 \mu \mathrm{m}$ ) and a high-magnification objective (Nikon CFI LU Plan APO EPI 150X, NA=0.95) with a 4X magnifier and a 300 $\mathrm{mm}$ extension tube for additional magnification. The actual magnification factor is calibrated to be $\sim 1300 \mathrm{X}$, corresponding to the image pixel size of $5 \mathrm{~nm} \times 5 \mathrm{~nm}$. Polarizer (P2) is inserted into the optical path before the camera to select the desired detection polarization $\left(E_{y}\right)$. The field maps were obtained by $z$-scanning with a step size of $5 \mathrm{~nm}$ using a piezo stage (PI E517) to precisely locate phase singularities. Another piezo stage (Nano-LP200, Mad City Labs) with nominal resolution of $0.4 \mathrm{~nm}$ was used for scanning in $x$-direction in the metrology experiment.

\section{Design of the interferometry metasurface}

The 1D Pancharatnam-Berry phase metasurface is fabricated in a $100 \mathrm{~nm}$-thickness gold film with focused ion beam milling with slits that are $400 \mathrm{~nm}$ long and $50 \mathrm{~nm}$ wide (the unit cell size is $400 \mathrm{~nm} \times 400 \mathrm{~nm}$ ). It creates superoscillatory focus at $\sim 10 \mu \mathrm{m}$ from the metasurface which contains rows of identical sub-wavelength slits oriented at either $+45^{\circ}$ or $-45^{\circ}$ with respect to the $x$-axis and has translation symmetry in the $y$ dimension. The mask is designed to be polarization-sensitive and creates a superoscillatory field only in cross-polarization to the incident wave. 
The pattern of the metasurface is designed using the binary particle swarm optimization algorithm (16). It optimizes a problem with regard to a given merit function using a population of 'particles' in the $N$-dimensional search space. Firstly, the transverse direction (assuming $x$ ) is divided into $N$ pair of equally spaced slits which are symmetric with respect to the geometrical center $(x=0)$. Each slit has either $\pi$ (' 1 ') or zero ('0') phase retardation value which corresponds to the slits orientating in $+45^{\circ}$ and $-45^{\circ}$ respectively. The algorithm searches for the best arrangement of these binary values. The target function is defined as

$$
I^{\operatorname{tar}}(x, z)=[\operatorname{sinc}(a x)]^{2} \exp \left[-\frac{\left(z-z_{f}\right)^{2}}{b^{2}}\right]
$$

where $\operatorname{sinc}(\cdot)$ is the sinc function to describe the field profile in the transverse plane, $z_{f}$ is the desired focal length, $a=\frac{0.886}{\mathrm{FWHM}}, b=\frac{\mathrm{DOF}}{2 \sqrt{\ln 2}}$, FWHM is the full-width half maximum of transverse superoscillatory hotspot size and DOF is the depth of focus. The merit function is given as

$$
F(r, z)=\left|I^{a c t}(x, z)-I^{\text {tar }}(x, z)\right|^{2}
$$

where $I^{a c t}(x, z)$ is the normalized actual intensity distribution and is calculated by angular spectrum method for certain mask design. The optimal mask is achieved after sufficient iterations until $F(r, z)$ gets to minimum.

The 2D random Pancharatnam-Berry phase metasurface is fabricated in a similar manner and contains a square grid of randomly oriented slits at either $+45^{\circ}$ or $-45^{\circ}$ orientation. The unit cell size is $300 \mathrm{~nm} \times 300 \mathrm{~nm}$. Each slit length is $200 \mathrm{~nm}$ long and $60 \mathrm{~nm}$ wide. 


\section{Phase retrieval}

The intensity and phase retrieval of the superoscillatory field starts from mapping the intensity profile of the $y$-polarized field. If the metasurface is illuminated with light with $+45^{\circ}-45^{\circ}$ linear polarization, and right/left circular polarization (RCP/LCP), the phase distributions of the $y$-polarized component of the field $\varphi(x, y)=\arg \left(E_{y}\right)$ can be retrieved from the intensity distribution of this component $I_{y}(x, y)$ of the interference pattern at a distance $z$ from the mask.

Indeed, the metamaterial mask works as a binary phase grating and produces a superoscillatory field in $E_{y}^{x}$ and a plane wave in $E_{x}^{x}$ under $x$-polarization excitation. Here the superscripts and subscripts denote polarization of the incident field and detected field respectively. If illuminated by left-handed circularly polarized ('LCP') light, the transmitted field can be expressed as

$$
E_{y}^{L C P}=\frac{1}{\sqrt{2}}\left(E_{y}^{x}+i E_{y}^{y}\right)
$$

and the corresponding intensity is given by

$$
I_{y}^{L C P}=\left(I_{y}^{x}+I_{y}^{y}\right) / 2+\sqrt{I_{y}^{x} \cdot I_{y}^{y}} \sin \delta
$$

where $\delta=\varphi-\varphi_{P W}$ is the phase difference between the superoscillatory field $E_{y}^{x}$ $\left(\varphi=\arg \left(E_{y}^{x}\right)\right)$ and the plane wave $E_{y}^{y}\left(\varphi_{P W}=k_{0} z\right)$. Similarly, for right-handed circularly polarized light ('RCP')

$$
I_{y}^{R C P}=\left(I_{y}^{x}+I_{y}^{y}\right) / 2-\sqrt{I_{y}^{x} \cdot I_{y}^{y}} \sin \delta,
$$


And for incident linear polarizations at $+45^{\circ}$ and $-45^{\circ}$

$$
I_{y}^{ \pm 45^{\circ}}=\left(I_{y}^{x}+I_{y}^{y}\right) / 2 \pm \sqrt{I_{y}^{x} \cdot I_{y}^{y}} \cos \delta
$$

From equations (s3)-(s6), we derive that:

$$
\varphi=\operatorname{atan}\left(\frac{I_{y}^{L C P}-I_{y}^{R C P}}{I_{y}^{+45^{\circ}}-I_{y}^{-45^{\circ}}}\right)+k_{0} z
$$

Therefore, by measuring intensity maps $\left(I_{y}^{L C P}, I_{y}^{R C P}, I_{y}^{+45^{\circ}}, I_{y}^{-45^{\circ}}\right)$, we are able to retrieve the phase of the superoscillatory field. The transverse local wavevector can then be calculated as $k_{x}=\nabla_{x} \varphi$.

\section{Detector's noise and resolving power}

The zones of high phase gradients are located in the areas of low intensity. However, the four intensity components used for phase retrieval in formula (s7) are not zero due to the contribution of plane wave in the cross-polarization. To evaluate the role of noise on the accuracy of recovering the reference mark's position of the optical ruler, we modelled maps of the local wavevector at different levels of noise at the detector (see Fig. S2). Here, the relative noise level is defined as the ratio of the noise power at the detector to the detected optical power level. The increase of the noise level from $0 \%$ to $20 \%$ results in a steady decrease of resolving power from $190 \mathrm{pm}(\lambda / 4200)$ to $810 \mathrm{pm}(\lambda / 990), 1.1 \mathrm{~nm}$ $(\lambda / 730)$ and $1.5 \mathrm{~nm}(\lambda / 530)$ respectively, as evaluated from the autocorrelation function. 


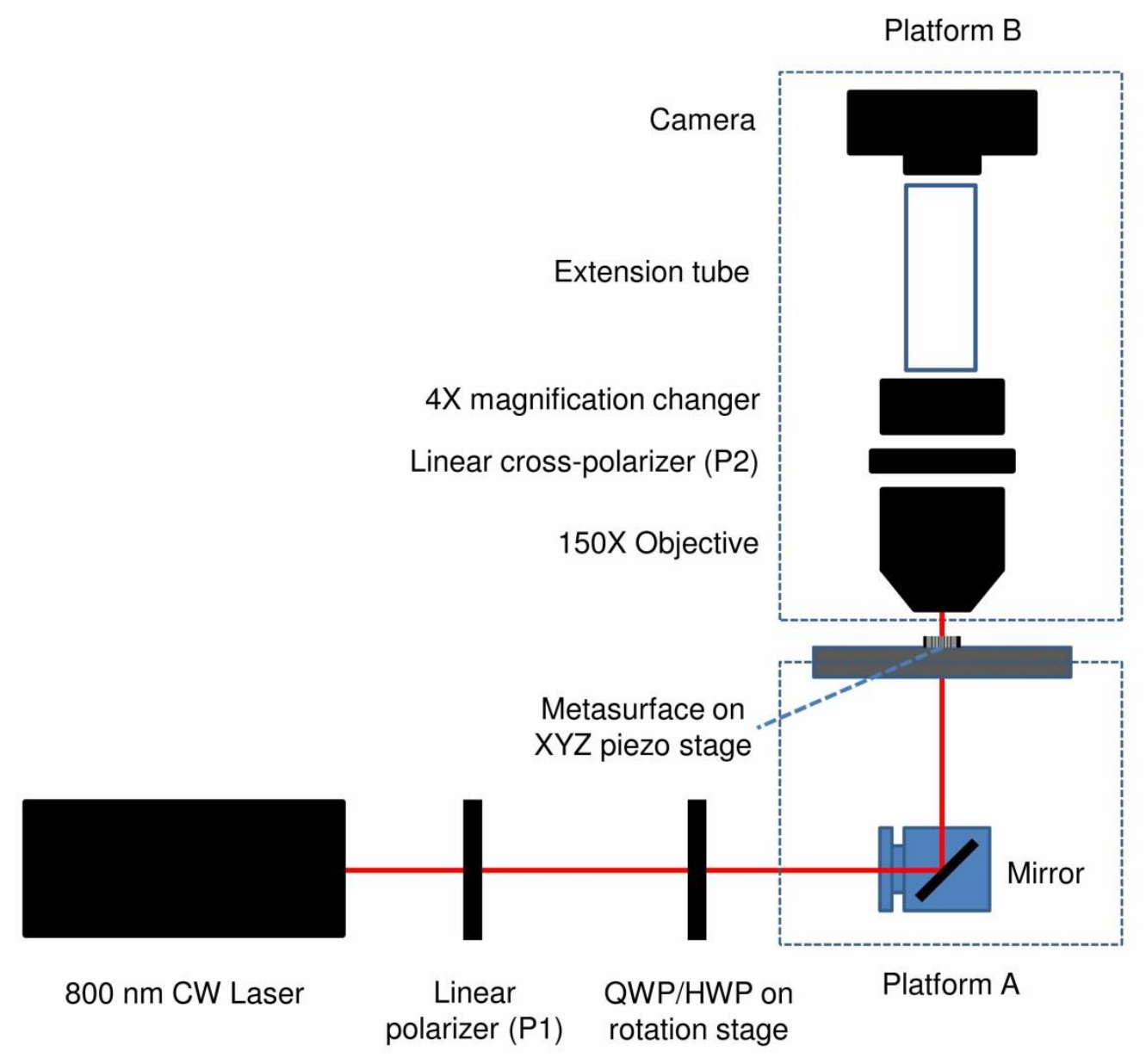

Fig. S1. Optical ruler metrology. Experimental setup for proof-of-principle demonstration. 


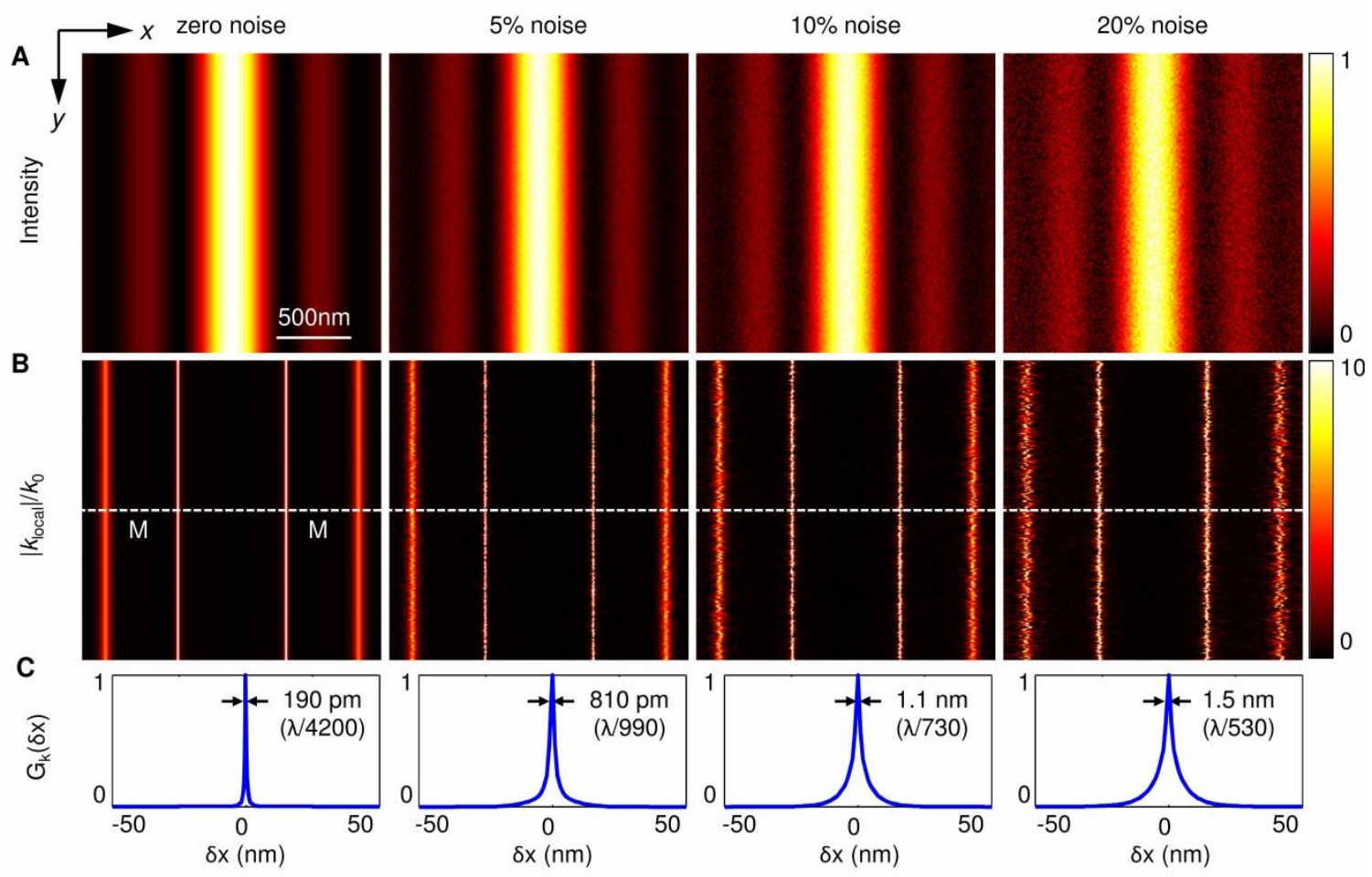

Fig. S2. Effect of detector's noise on retrieval of the reference marks of the optical ruler. (A) Intensity maps; (B) Local wavevector distributions; (C) Autocorrelation functions of wavevector maps. The $1^{\text {st }}, 2^{\text {nd }}, 3^{\text {rd }}$ and $4^{\text {th }}$ columns correspond to a noise level of $0 \%, 5 \%, 10 \%$, and $20 \%$ respectively. 